composent la population scolaire de l'Union française, un lien intellectuel en même temps que le sentiment de l'appartenance à une même communauté.'

Au total huit élèves de la métropole ont été envoyés dans un territoire d'outre-mer et quinze sont venus de ces pays dans la métropole. Leur nombre sera plus important cette année comme sera plus important le nombre de jeunes gens qui aujourd'hui réfléchissent sur les problèmes posés par l'édification de cette vaste communauté qu'est l'Union française.

A Paris, la semaine a commencé par une conférence à la Sorbonne, prononcée par M. Hubert Deschamps, Directeur adjoint de la recherche scientifique à la France d'Outre-mer, à l'intention des professeurs. Le i $\varsigma$ mars, au Musée pédagogique, des délégations des lycées, collèges et écoles de Paris ont assisté à une conférence de Mme Malroux, suivie de la projection du film 'Paysans Noirs'.

\title{
Un Centre permanent d'Art africain
}

UN centre artisanal africain a été ouvert à Brazzaville où seront offerts non seulement les œuvres des étudiants et artisans de l'École des Arts et de l'artisanat de Brazzaville, mais également les produits de l'artisanat populaire spontané, particulièrement riche au Tchad.

L'École d'Art du Moyen-Congo en sera l'un des principaux exposants et fournisseurs, mais on parle également d'un important arrivage d'objets du 'Tchad et spécialement de très beaux tapis. Cette réalisation n'a été possible que grâce à l'effort accompli par l'École d'Art de Brazzaville. L'École d'Art compte actuellement 33 élèves, répartis en trois années, soit I I élèves par promotion. Elle comprend trois sections : une section de reliure et de maroquinerie, une section de sculpture sur bois et ivoire, une section de céramique-poterie.

Les ateliers, les tours et les fours de l'école sont en outre mis à la disposition d'une coopérative d'artisans anciens élèves qui comprend $\mathrm{I} 3$ associés, dont 9 sont céramistes. On s'est efforcé depuis la création de ce centre de respecter l'inspiration et le style propre de l'art africain, en aidant surtout ses élèves à améliorer leur technique et la qualité de leur production, en les défendant contre les facilités et plus encore contre les tentations du mercantilisme.

\section{Le développement des Sciences sociales et le Congo Belge}

L'importance d'une investigation méthodique en Afrique dans le champ des sciences sociales est admise partout. Dans le Congo Belge, l'Institut pour la recherche scientifique en Afrique Centrale, hautement conçu, a entrepris pendant plusieurs années une œuvre appréciée dans ce domaine. D'autres initiatives des Universités belges sont en train de se faire jour. L'Institut de Sociologie de l'Université de Liège entreprend d'établir un centre de recherches à Élisabethville, grâce à la collaboration du Centre d'études des problèmes sociaux indigènes (C.E.P.S.I.) et au bienveillant appui du Ministre des Colonies. Le développement de ce Centre, qui constituera une contribution de l'Université à l'œuvre africaine dans le domaine des sciences sociales, servira du même coup le développement des études sociologiques à Liège.

\section{Institute of Social and Economic Research, Rhodes University}

Mr. M. WILkEs has begun a two years' study of the conservative rural Xhosa (' red blanket people ') as part of the Border Regional Survey (see Africa, April 1956, p. 188). He is at present engaged on an intensive field-study among a rural community in the King Williamstown District.

Dr. D. H. Reader is entering the second stage of his field-work among urban Xhosa in the Native Locations of East London, and is collecting and analysing quantitative data with the help of African assistants. 